\title{
Correction to: Ratcheting Response of SS316 Steel Samples with Different Notch Shapes under Various Loading Spectra
}

\author{
A. Shekarian and A. Varvani-Farahani (D)
}

\section{Correction to: JMEPEG https://doi.org/10.1007/s11665-021-05639-x}

As a result of an error during the publication process, a number of equations were displayed incorrectly in the PDF version of this article as originally published.

The following shows the incorrect and correct presentations of the equations in question:

Mistyped equation:

$d-\alpha=\sum_{i=1}^{M} d-\alpha_{i}, d-\alpha_{i}=\frac{2}{3} C_{i} d-\varepsilon_{P}-\gamma_{i}^{\prime}-\alpha_{i} d p$

Correct equation:

$d \bar{\alpha}=\sum_{i=1}^{M} d \bar{\alpha}_{i}, d \bar{\alpha}_{i}=\frac{2}{3} C_{i} d \bar{\varepsilon}_{P}-\gamma_{i}^{\prime} \bar{\alpha}_{i} d p$

Mistyped equation:

$d p=\sqrt{\frac{2}{3} d-\varepsilon_{P} \cdot d-\varepsilon_{P}}$

Correct equation:

$d p=\sqrt{\frac{2}{3} d \bar{\varepsilon}_{P} \cdot d \bar{\varepsilon}_{P}}$

Mistyped equation:

$d-\alpha=C d-\varepsilon_{p}-\gamma_{1}(-\alpha-\delta-b) d p$
Correct equation:

$d \bar{\alpha}=C d \bar{\varepsilon}_{p}-\gamma_{1}(\bar{\alpha}-\delta \bar{b}) d p$

Mistyped equation:

$d-b=\gamma_{2}(-\alpha--b) d p$

Correct equation:

$d \bar{b}=\gamma_{2}(\bar{\alpha}-\bar{b}) d p$

The term $-\varepsilon_{P}$ should be $\bar{\varepsilon}_{P}$

The term $-b$ should be $\bar{b}$

The term $(-\alpha-\delta-b)$ should be $(\bar{\alpha}-\delta \bar{b})$

The term $\sum_{i=1}^{M} d-\alpha_{i}$ should be $\sum_{i=1}^{M} d \bar{\alpha}_{i}$

The term $d-\varepsilon_{p}$ should be $d \bar{\varepsilon}_{p}$

The term $\sum_{i=1}^{M} d-\alpha_{i}$ should be $\sum_{i=1}^{M} d \bar{\alpha}_{i}$

The term $(-\alpha-\delta-b)$ should be $(\bar{\alpha}-\delta \bar{b})$

Under section "2.2 The Ahmadzadeh-Varvani (A-V) Kinematic Hardening Rule" of the published manuscript, terms $\delta$ and $k$ in the below lines are mistyped as shown in the following text:

$\delta=(l e x \alpha /-l e x k)^{m}$. Constant $k$ is the ratio of coefficients $C$ and $\gamma_{1}$ as $k=\operatorname{lex} C /-l e x \gamma_{1}$, and exponent $m$ is a material

The corrected terms $\delta$ and $k$ within the text should be given as below:

$\delta=(\alpha / k)^{m}$. Constant $k$ is the ratio of coefficients $C$ and $\gamma_{1}$ as $k=C / \gamma_{1}$, and exponent $m$ is a material constant.

The original article has been corrected.

Publisher's Note Springer Nature remains neutral with regard to jurisdictional claims in published maps and institutional affiliations.

The original article can be found online at https://doi.org/10.1007/ s11665-021-05639-x.

A. Shekarian and A. Varvani-Farahani, Department of Mechanical and Industrial Engineering, Ryerson University, 350 Victoria Street, Toronto, Ontario M5B 2K3, Canada. Contact e-mail: avarvani@ryerson.ca. 